\title{
Le lussazioni della pelvi
}

\author{
Merildo Maritato, Giovanni Benellia $(\bowtie)$ \\ U.O. Ortopedia, Azienda UsI Toscana Centro, Prato, Italia \\ agiovanni.benelli@uslcentro.toscana.it
}

\begin{abstract}
Pelvic dislocation
Pelvic dislocation is a rare event. In the English-language literature only one case report has been reported. In this article we discuss the traumatic and post-partum dislocation of the pelvic joint, the modality of occurrence and the possible treatment
\end{abstract}

Pubblicato online: 24 luglio 2018

(c) The Author(s) 2018

La pelvi è un osso che congiunge il tronco e la colonna vertebrale agli arti inferiori, con forma di bacinella, da cui il nome di bacino.

Nell'adulto il bacino risulta formato dal sacro e da due ossa innominate, dette anche emipelvi (Fig. 1).

L'osso innominato si forma dalla fusione dei tre segmenti ileo, ischio e pube che si riuniscono nella zona triradiata: la zona che nell'adulto corrisponderà all'articolazione.

Il complesso della pelvi presenta tre articolazioni proprie: la sacrolilaca destra, quella sinistra e la sinfisi pubica. Due articolazioni la collegano con l'arto inferiore: la coxofemorale destra e quella sinistra.

\section{Le articolazioni sacroliache}

La superficie articolare del sacro ha una forma a J, con apice anteriore e base posteriore, ed è concava; la superfice dell'ileo è invece convessa e si adatta a quella del sacro.

L'articolazione è una cavità virtuale, delimitata posteriormente dal legamento interosseo e anteriormente dal legamento sacro-iliaco anteriore. Dietro al legamento interosseo si trova il legamento sacroiliaco posteriore, che non è proprio dell'articolazione ma è un legamento pelvico.

L'articolazione possiede, inoltre, tre legamenti estrinseci:

- il legamento ileolombare, che si tende tra l'apice del processo costiforme della quinta vertebra lombare e la spina iliaca posteriore superiore. Offre l'inserzione al muscolo quadrato dei lombi

- il legamento sacrospinoso, che origina dal margine laterale dell'ala del sacro e si inserisce alla spina ischiatica. Esso contribuisce a trasformare la grande incisura ischiatica nel grande foro ischiatico

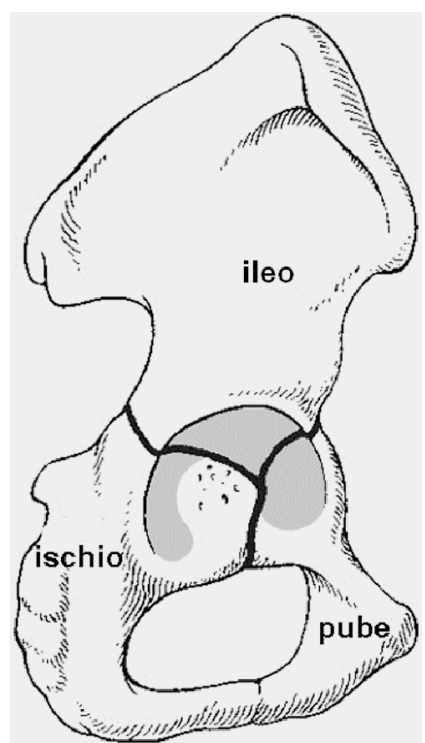

Fig. 1 - Osso innominato o emipelvi

- il legamento sacrotuberoso, che origina dal margine posteriore dell'osso iliaco, nella porzione compresa tra le spine iliache posteriori, e dal margine laterale dell'ala del sacro per inserirsi alla tuberosità ischiatica. Esso contribuisce, assieme al legamento sacrospinoso, a trasformare la piccola incisura ischiatica nel piccolo foro ischiatico.

Il legamento sacrotuberoso e il legamento sacrospinoso costituiscono, insieme, il legamento sacroischiatico.

\section{La sinfisi pubica}

Anteriormente il bacino viene chiuso dalle due ossa del pube che si articolano tra loro formando la sinfisi. 

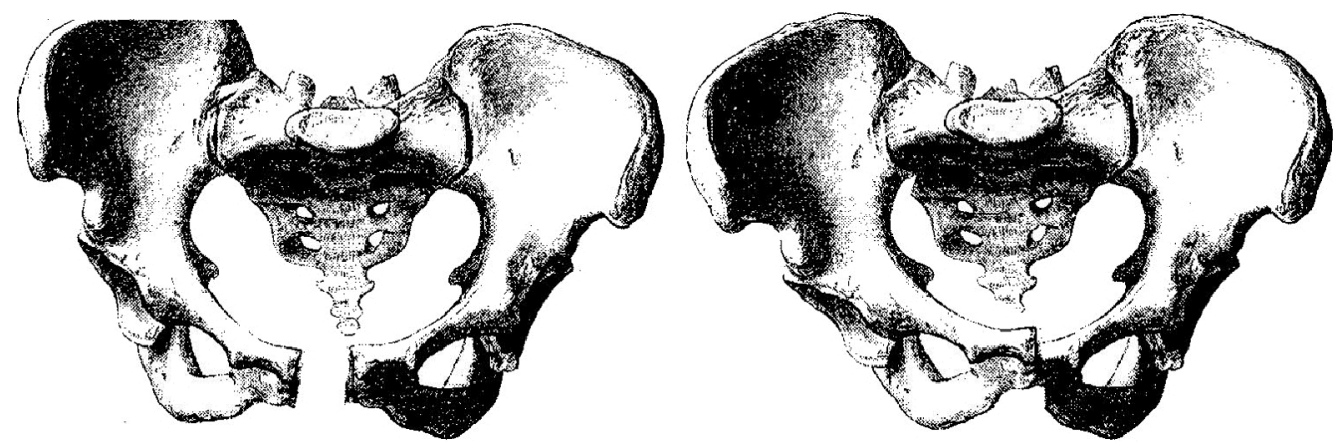

Fig. 2 - Lussazioni della sinfisi: in apertura e in chiusura

La sinfisi è un'articolazione trapezoidale più larga in alto e più stretta in basso, con una cavità articolare virtuale. Qui si interpone il menisco che, strutturalmente, assomiglia a un disco intervertebrale, più denso nella regione periferica e molliccio al centro, ove l'articolazione si risolve in un'esile fessura. A cavallo della sinfisi troviamo il legamento arcuato del pube, smusso e arrotondato superiormente, esile e tagliente inferiormente.

I movimenti sulle articolazioni sacroliache e sulla sinfisi, sono:

- la nutazione: la base del sacro si sposta in basso e in avanti, mentre l'apice si sposta indietro. Lo stretto superiore del bacino diminuisce di diametro, lo stretto inferiore aumenta la sua ampiezza. L'antiversione del bacino si accompagna al movimento di nutazione del sacro

- la contronutazione: la base del sacro si sposta in alto e indietro, mentre l'apice si sposta in avanti e in basso. Lo stretto superiore del bacino aumenta di diametro, lo stretto inferiore diminuisce la sua ampiezza. La retroversione del bacino si accompagna al movimento di contronutazione del sacro.

Questi movimenti sono indispensabili nella pelvi femminile durante le fasi del parto.

La prima pubblicazione in cui vengono descritte le lussazioni della pelvi risale al 1842 [1]. Nell'articolo, l'autore Astley Cooper cita: "Casi in cui l'osso innominato viene separato dal sacro e la sinfisi viene interrotta e fratturata [...] come nel caso 73, in cui la tremenda violenza del trauma danneggia le parti molli del bacino e i visceri vicini”. Nel 1847 Malgaigne, nel Traité des fractures e des luxations, descrive la lussazione superiore della pelvi, la frattura di Malgaigne [2]. Nell'aprile 1938, Watson-Jones pubblica sul BJS Dislocations and fracture-dislocations of the pelvis [3]. A ottobre del 1942, poi, sul BJS Taylor scrive un articolo sulla pelvic dislocation basato anche sulle esperienze di Cooper e Watson-Jones [4]. Nel 1948, Holdsworth pubblica sul JBJS (Br) Dislocation and fracture-dislocation of the pelvis, dove analizza il meccanismo di lesione [5].

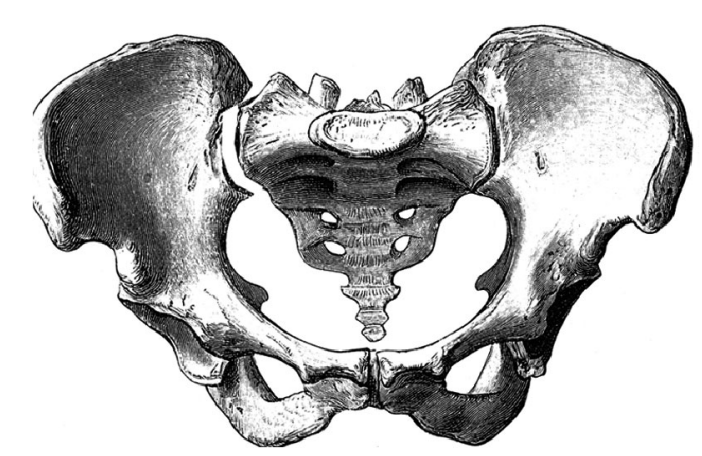

Fig. 3 - Lussazione isolata della sacroiliaca: in direzione anteriore 0 posteriore

Nel 1975 viene analizzato da Monahan il meccanismo lesionale e vengono citate le lussazioni della pelvi [6]. Nel 1980, Pennal e Tile su CORR 151 descrivono per primi la classificazione delle fatture e fratture-lussazioni [7].

Dai primi anni '80, con l'utilizzo estensivo della TC, la lussaziona della pelvi come entità a sé stante si è ridotta di frequenza.

L'incidenza delle fratture lussazioni della pelvi è rara e oscilla dal 2 al $4 \%$ di tutte le fratture.

Le lussazioni pure delle articolazioni della pelvi sono molto rare e in letteratura sono riportati solo dei case report con due classificazioni: quella di Zhang [8] e quella di Maqungo [9]. Occorrono quasi esclusivamente nei soggetti giovani in cui la resistenza meccanica dell'osso e la lassità articolare e legamentosa sono maggiori.

Le lussazione pure della pelvi sono classificabili in:

- lussazione isolata della sinfisi (Fig. 2)

- lussazione isolata della sacroiliaca (Fig. 3)

- lussazione combinata della sinfisi e della sacroiliaca (Fig. 4).

La sinfisi si può lussare in apertura o in chiusura con overlapping anteroposteriore: 

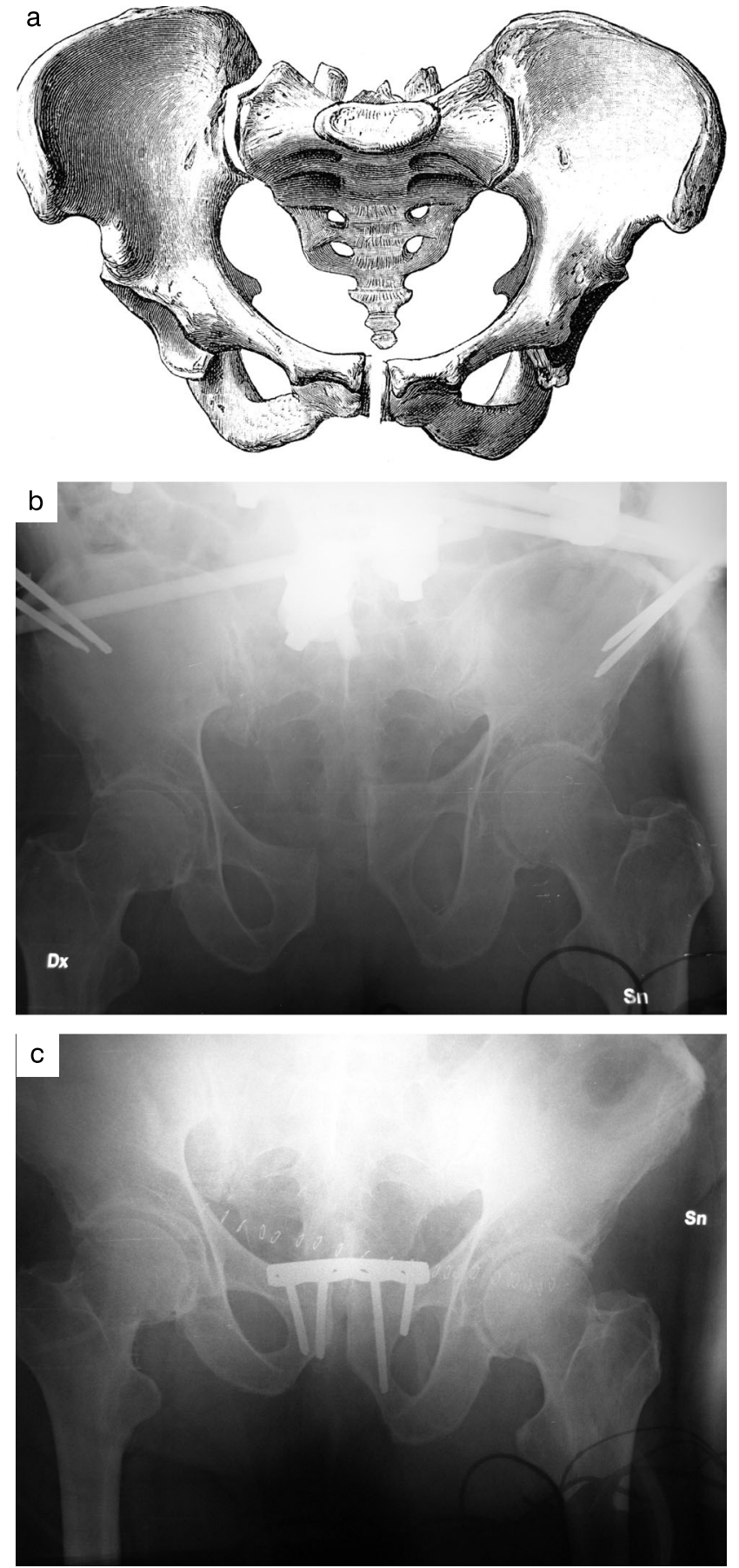

Fig. 4 - (a)-(c) Lussazione combinata: sacroiliaca e sinfisi

- la sacroiliaca si può dislocare in apertura, mantenendo una cerniera posteriore

- può dislocarsi completamente in alto nelle combinate

- può dislocarsi posteriormente o anteriormente

- può dislocarsi in "chiusura" anteriore (crush della porzione articolare anteriore, sacroiliac buckling) con apertura posteriore, in genere modesta.

I traumi che determinano le fratture-lussazioni determinano con frequenza molto più bassa le lussazioni pure (Table 1).

\section{Lussazioni della sinfisi pubica}

Il trauma in Antero-Posteriore (AP) è detto open book (lussazione in apertura della sinfisi), con progressiva apertura anche dell'articolazione sacroiliaca a partire dalla regione anteriore (Fig. 5). Presenta instabilità relativa, mentre si mantiene la cerniera posteriore; deve essere chiusa se l'apertura è consistente, attraverso manovre esterne (pelvic binding) o chirurgiche (sintesi interna o esterna).

La lesione vascolare posteriore può essere molto ampia; in genere è venosa e la chiusura dell'anello, riducendo il volume della pelvi, favorisce la riduzione del sanguinamento per collabimento delle strutture venose; raramente possono concomitare lesioni nervose, di L5, del nervo gluteo superiore.

Le lesioni arteriose sono rare e necessitano di tamponamento pelvico, di embolizzazione o di altra chiusura chirurgica. Le lesioni con apertura della sinfisi $>2,5 \mathrm{~cm}$ determinano un lesione posteriore significativa e sono chirurgiche.

Il trattamento chirurgico delle lussazioni pure della sinfisi pubica è la riduzione aperta e la stabilizzazione con placche; la via di accesso, in genere, è la Pfannestiel: la riduzione avviene attraverso Weber clamp o Faraboeuf clamp o Jungbluth clamp. Ognuna di esse è idonea poiché la lussazione deve essere solo chiusa. L'osso appartiene a soggetti giovani e la presa è buona con ogni strumento.

Con una deviazione assiale importante occorrono due strumenti per mantenere la riduzione sui due assi di traslazione. In genere, una pinza di Faraboeuf o Jungbluth e una Weber; la vite di ancoraggio delle pinze da riduzione deve avere la posizione frontale per permettere il posizionamento della placca e delle due viti di mantenimento iniziale.

La placca deve essere da 3,5 con un minimo di 4 fori (meglio di 6), ma sempre in numero pari, altrimenti si rende una placca da sinfisi. Talvolta è necessaria una doppia placca ortogonale (Fig. 4b,c).

I traumi in compressione laterale sono detti bucket handle; determinano lussazioni della sinfisi in chiusura e rappresentano una lesione stabile (Fig. 6).

Le lussazioni pure in chiusura della sinfisi sono rappresentate dalla overlapped symphysis o dalla locked symphysis. Sono eventi rari che avvengono per traumi in compressione laterale; in genere sono associate fratture della regione anteriore della sacroiliaca o dell'ala sacrale, che vengono diagnosticate con la solo TC perché quasi sempre non visibili direttamente con RX.

Presentano frequenti rischi lesionali nel maschio (uretra, prostata, plesso venoso prostatico); con una certa frequenza esitano in insufficienza erettile. 
Tabella 1 Classificazione delle lussazioni pelviche pure, meccanismo traumatico

\begin{tabular}{llllll}
\hline Lussazione sinfisi & Trauma & Lussazione sacroiliaca & Trauma & Lussazione combinata & Trauma \\
\hline Apertura & Open book & $\begin{array}{l}\text { Apertura anteriore e } \\
\text { chiusura posteriore }\end{array}$ & $\begin{array}{l}\text { Open book } \\
\text { AP compression }\end{array}$ & $\begin{array}{l}\text { Sacroiliaca e sinfisi } \\
\text { anteriori o posteriori }\end{array}$ & $\begin{array}{l}\text { AP compression o } \\
\text { vertical shear }\end{array}$ \\
$\begin{array}{l}\text { A compression } \\
\text { anteriore/posteriore }\end{array}$ & Lateral compression & Posteriore/superiore & AP compression & $\begin{array}{l}\text { Sacroiliaca e sinfisi } \\
\text { superiori }\end{array}$ & Vertical shear \\
\hline
\end{tabular}

Fig. 5 - Open book, trauma in AP compression, aperture sinfisi pubica e sacroiliaca posteriore

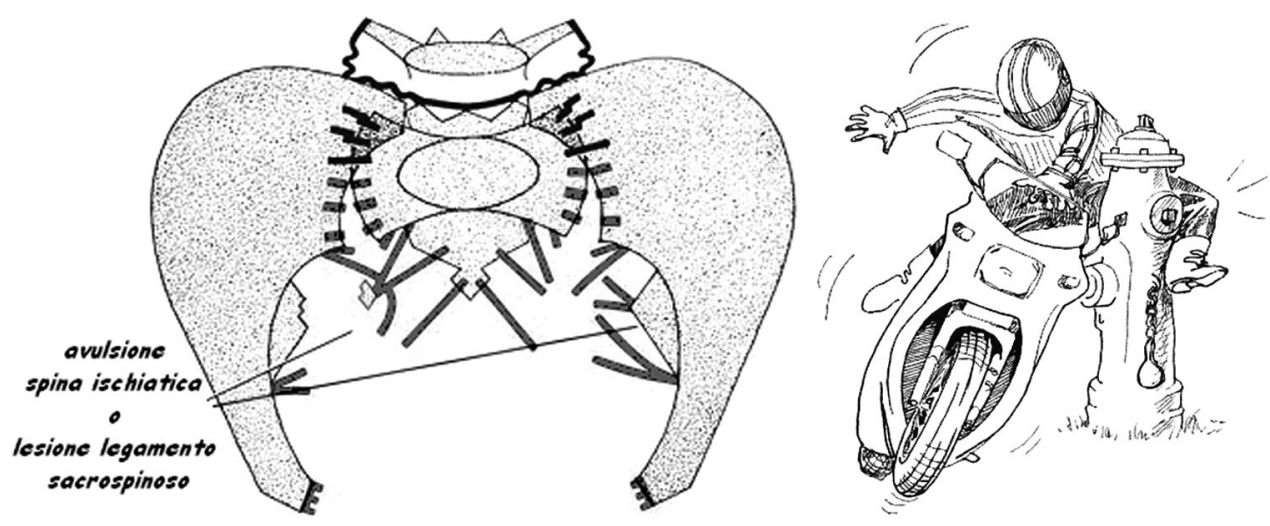

Fig. 6 - Bucket handle, trauma in lateral compression, chiusura della sinfisi pubica, locked symphysis, compressione anteriore della sacroiliaca (sacroiliac buckling)
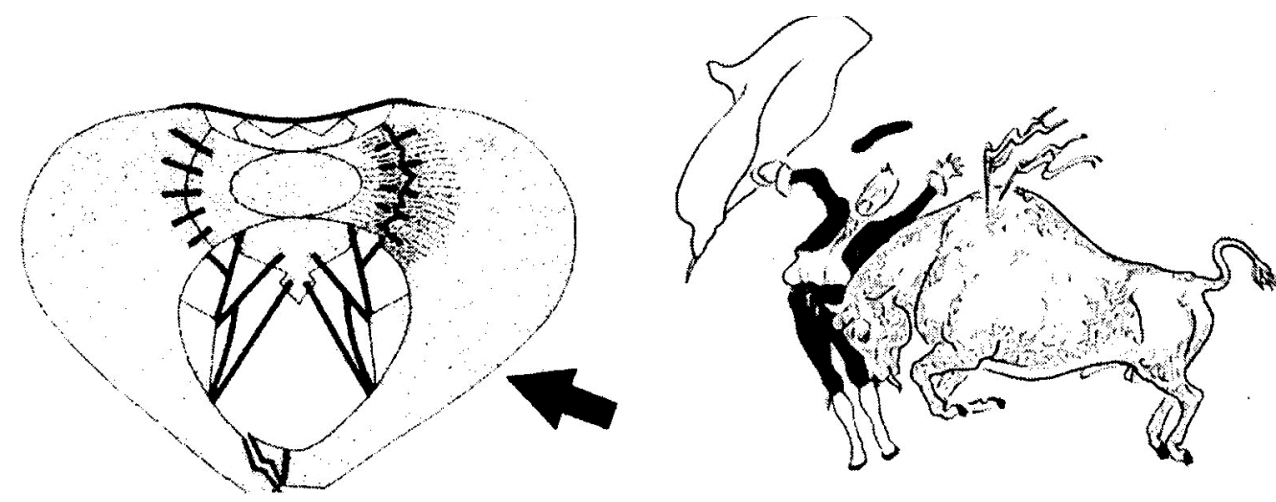

In letteratura esistono molti case report al riguardo [9-15]. Maqungo propone una classificazione in tre gradi di gravità crescente; il $2^{\circ}$ e il $3^{\circ}$ grado richiedono sempre un trattamento chirurgico. Sono lussazioni tipiche dei maschi perché, come suggerito da Cannada e Reinert [12], la sinfisi delle donne ha un disco più spesso e una mobilità maggiore.

Il trattamento è chirurgico, con accesso di Pfannestiel e osteosintesi interna. La riduzione viene eseguita nel grado 2 con leva da osso o cobb elevator nella zona di overlapping, aiutandosi con uno strumento di riduzione.

In genere viene utilizzata la Jungbluth clamp perché può muoversi nelle tre direzioni dello spazio: in apertura, in chiusura, in traslazione anteriore e posteriore, e in décalage superiore e inferiore.

La vite di ancoraggio della clamp deve essere lunga e posizio- nata sulla faccia anteriore dei due rami pubici destro e sinistro a distanza di circa 5-6 cm per permettere il disingaggio e la traslazione.

La riduzione viene poi mantenuta manualmente o tramite compressione della vite di serraggio della Jungbluth. L'osteosintesi viene eseguita con placca 3,5 a fori pari (meglio sei), o con placca da sinfisi dedicata.

Nelle locked symphysis incarcerate nel forame otturatorio può essere necessaria osteotomia dei rami pubici con accesso intrapelvico e sintesi più ampia con placca passante l'osteotomia [14].

\section{Lussazioni combinate sinfisi-sacroiliaca}

Il trauma da taglio verticale è detto vertical shear; è una lussazione tipica della caduta dall'alto. 

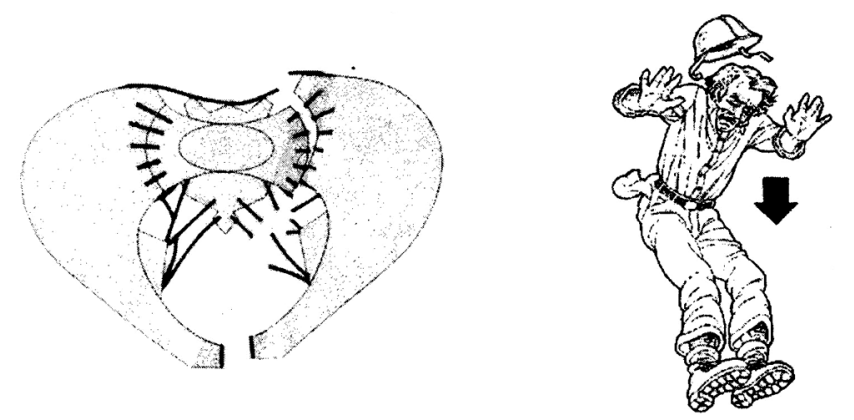

Fig. 7 - Vertical shear, trauma di taglio progressivo, lesione sinfisi e sacroiliaca

Il trauma transita dalla sinfisi verso un'articolazione sacroiliaca, con un progressivo pattern lesionale (Fig. 7).

Occorre far attenzione alla possibilità che alle RX le due emipelvi siano ambedue congruenti sul sacro ma instabili: occorrono delle gentili manovre di trazione per valutare se ci sia instabilità e confermare la diagnosi con TC. In genere, la dislocazione dell'articolazione sacroiliaca è posteriore $\mathrm{e}$ verso l'alto. Possono coesistere lesioni vascolo-nervose, a livello di L4, L5, del nervo gluteo superiore, del plesso venoso posteriore, dell'arteria glutea superiore, del plesso venoso prostatico e dell'innervazione perineale.

Può essere necessaria la stabilizzazione in urgenza, con fissazione esterna (pelvic clamp). Dato che la lesione è tutta aggredibile da un accesso anteriore, l'osteosintesi è in genere anteriore.

Le vie di accesso che possono essere eseguite sono tre: la ileoinguinale, anche mininvasiva (prima e terza finestra), la Stoppa classica e la Stoppa modificata (midline). Tutte giungono sulle due articolazioni dal versante anteriore.

La prima articolazione da ridurre è la sacroiliaca. In genere, nell'ileoinguinale viene ridotta manualmente trazionando con Faraboeuf clamp sull'ala iliaca e stabilizzando con $\mathrm{Fa}$ raboeuf o Jungbluth ancorato a due viti, sacrale e iliaca, ai due lati della sacroiliaca.

Nella Stoppa è più comodo usare la Jungbluth con ancoraggio sacrale e iliaco; la pinza deve essere lunga e sovente risultano necessarie due viti da 4,5 (tipiche della pinza lunga).

La stabilizzazione della sacroiliaca viene eseguita con doppia placca da 3,5 a due, tre o quattro fori, in posizione ortogonale l'una rispetto all'altra.

Solo dopo occorrerà perfezionare la riduzione della sinfisi ed eseguire un'osteosintesi con placca anteriore sinfisaria.

Talvolta può essere indicato un accesso di Pfannesteil per l'osteosintesi sinfisaria e viti transacrali o una placca posteriore sagomata a $U$.

\section{Le lussazioni isolate della sacroiliaca}

Sono eventi rarissimi, tipici nei bambini.
Nel 1976 Lewis e Arnold [16] descrivono un caso di lussazione anteriore della sacroiliaca in un bambino; tuttavia, in questo caso era associata la frattura dell'osso innominato omolaterale, sul fondo acetabolare. Queste lussazioni sono state ben descritte in letteratura da Zhang, che propone una classificazione in anteriori e posteriori [8]. Nel 2012, Bouguennec et al. descrivono un caso in un paziente di 20 anni [17].

Esistono, inoltre, nella letteratura anglosassone alcuni case report di lussazioni bilaterali con anello integro $[18,19]$. Anch'esse sono eventi rarissimi, associati spesso a lesioni di plesso con più o meno gravi lesioni vascolari; la riduzione è pressoché sempre chirurgica. Questi traumi si verificano in pazienti molto giovani, bambini, in cui il trattamento è in genere con fissazione esterna.

Talora è necessaria una riduzione cruenta della lussazione, che viene eseguita trazionando l'ala iliaca posteriore, vicino all'articolazione lussata, tramite una piccola incisione e il posizionamento di una pinza di Faraboeuf che servirà come supporto di trazione.

Un capitolo a parte sono le lussazioni in concomitanza di un parto naturale. Durante il parto la regione della sinfisi pubica, ma anche le sacroiliache, in relazione alla lassità legamentosa acquisita durante la gravidanza, diventano molto più elastiche. Le pazienti in gravidanza presentano un laxity test positivo, che ritorna come in precedenza dopo alcuni mesi dal parto [20].

La diastasi media post-partum è di 1,0-1,3 cm [21]; sono riportati in letteratura casi di diastasi molto maggiore, anche di 4-8 cm [22-24]; inoltre, in letteratura è riportato anche un case report di instabilità verticale completa post-partum. In genere, le diastasi della sinfisi di dimensioni minori, fino a $2 \mathrm{~cm}$ circa, tornano a una restitutio completa, tenuto conto che la lassità durante la gravidanza incrementa il movimento sinfisario naturale nella donna (in genere di 2-3 mm) a circa il doppio. Con diastasi maggiori di $2 \mathrm{~cm}$ c'è il rischio di una lesione anatomica menisco-capsulare tale da non garantire un ripristino dell'articolazione.

Talora può essere sufficiente un trattamento incruento (binder) di alcuni giorni (da 7 a 9). L'osteosintesi anteriore è indicata dopo conferma diagnostica (RMN) di lesione completa dell'articolazione sinfisaria, confermata anche dall'ematoma retropubico, che testimonia la rottura anatomica dell'articolazione.

Conflitto Di InTeresse Gli autori Merildo Maritato e Giovanni Benelli dichiarano di non avere alcun conflitto di interesse.

CONSENSO INFORMATO E CONFORMITÃ AGLI STANDARD ETICI Tutte le procedure descritte nello studio e che hanno coinvolto esseri umani sono state attuate in conformità alle norme etiche stabilite dalla dichiarazione di Helsinki del 1975 e successive modifiche. Il consenso informato è stato ottenuto da tutti i pazienti inclusi nello studio. 
Human and Animal Rights L'articolo non contiene alcuno studio eseguito su esseri umani e su animali da parte degli autori.

OPEN ACCESS This article is distributed under the terms of the Creative Commons Attribution 4.0 International License (http://creative commons.org/licenses/by/4.0/), which permits unrestricted use, distribution, and reproduction in any medium, provided you give appropriate credit to the original author(s) and the source, provide a link to the Creative Commons license, and indicate if changes were made.

\section{Bibliografia}

1. Cooper A (1842) A treatise on fractures and dislocations of the joints. Churchill, London

2. Malgaigne JF (1855) Traité des fractures e des luxation. Chez J.B. Baillière, Paris

3. Watson-Jones R (1938) Dislocations and fracture-dislocations of the pelvis. Br J Surg 25:773-781

4. Taylor RG (1942) Pelvic dislocation. Br J Surg 118:126-132

5. Holdsworth FW (1948) Dislocation and fracture-dislocation of the pelvis. J Bone Jt Surg, Br 30:461-466

6. Monahan PR, Taylor RG (1975) Dislocation and fracture dislocation of the pelvis. Injury 6(4):325-333

7. Pennal GF, Tile M, Waddell JP, Garside H (1980) Pelvic disruption: assessment and classification. Clin Orthop Relat Res 151:12-21

8. Zhang Q, Chen W, Liu H et al (2009) The anterior dislocation of the sacroiliac joint: a report of four cases and review of the literature and treatment algorism. Arch Orthop Trauma Surg 129:941-947

9. Maqungo S, Koller I, Roche S (2010) Overlapping pubic symphysis dislocation: a case report and proposal of a classification system. SA Orthop J 9(4):74-77

10. Tadros AM, Lunsjo K, O’Brien P (2009) Overlapping dislocation of the pubic symphysis: report of three cases and review of the literature. Arch Orthop Trauma Surg 129:679-683
11. Afshar A, Koushkzari M (2015) Overlapped pubic symphysis; a case report and review of the literature. Arch Bone Joint Surg 3(3):212-216

12. Cannada LK, Reinert CM (2009) Case report. Locked pubic symphysis - an open reduction technique. Clin Orthop Relat Res 467:2192-2195

13. Thulasiraman V, Ramesh Pandian TR, Ashok S (2010) Locked pubic symphysis—a case series. Injury Extra 41:20-24

14. Li KH, Sun BH, Zhu Y, Long HT (2013) Locked pubic symphysis into the obturator foramen: a rare case presentation and literature review. Orthop Traumatol, Surg Res 99:106-110

15. Sreesobh KV, Sageer AM, Raffic M (2006) Locked overlapping dislocation of the pubic symphysis into the obturator foramen: a case report. J Orthop Surg 14(2):200-203

16. Lewis M, Arnold WD (1976) Complete anterior dislocation of the sacro-iliac joint. J Bone Jt Surg, Am 58:136-138

17. Bouguennec N, Gouin F, Piétu G (2012) Case report. Isolated anterior unilateral sacroiliac dislocation without pubic arch disjunction. Orthop Traumatol, Surg Res 98:359-362

18. Loupasis G, Anastopoulos G, Asimakopoulos A (2005) Pure bilateral sacroiliac dislocation with intact anterior pelvis. Injury 36:1379-1382

19. Wright V, Zelle BA, Prayson M (2004) Bilateral sacroiliac joint dislocation without associated fracture or anterior pelvic ring injuries. J Orthop Trauma 18:634-637

20. Calguneri M, Bird HA, Wright V (1982) Changes in joint laxity occurring during pregnancy. Ann Rheum Dis 41:126-128

21. Parker JM, Bhattacharjee M (2009) Peripartum diastasis of the symphysis pubis. N Engl J Med 361:19

22. Hou Z, Riehl JT, Smith WR et al (2011) Severe postpartum disruption of the pelvic ring: report of two cases and review of the literature. Pat Saf Surg 5:2

23. Gräf C, Sellei RM, Schrading S, Bauerschlag DO (2014) Treatment of parturition-induced rupture of pubic symphysis after spontaneous vaginal delivery. Case Rep Obst Gynecol 2014:1-3

24. Pires RE, Labronici PJ, Giordano V et al (2015) Intrapartum pubic symphysis disruption. Ann Med Health Sci Res 5(6):476-479 